\title{
Nipple-sparing mastectomy for early breast cancer: the importance of intraoperative evaluation of retroareolar margins and intra-nipple duct removal
}

\author{
Rebeca Neves Heinzen ${ }^{1}$, Alfredo Carlos Simões Dornellas de Barros ${ }^{2}$, Filomena Marino Carvalho ${ }^{2}$, \\ Fernando Nalesso Aguiar ${ }^{2}$, Cristiane da Costa Bandeira Abrahão Nimir ${ }^{3}$, Alfredo Luiz Jacomo ${ }^{1}$ \\ ${ }^{1}$ Discipline of Human Structural Topography, ${ }^{2}$ Discipline of Pathologic Anatomy, University of São Paulo School of Medicine, São Paulo, Brazil; \\ ${ }^{3}$ Department of Breast Surgery, Federal University of São Paulo, São Paulo, Brazil \\ Contributions: (I) Conception and design: RN Heinzen, ACSD de Barros, AL Jacomo; (II) Administrative support: ACSD de Barros, AL Jacomo; (III) \\ Provision of study materials or patients: ACSD de Barros, FM Carvalho, FN Aguiar, CCBA Nimir; (IV) Collection and assembly data: RN Heinzen; \\ (V) Data analysis and interpretation: RN Heinzen, ACSD de Barros, AL Jacomo; (VI) Manuscript writing: All authors; (VII) Final approval of \\ manuscript: All authors. \\ Correspondence to: Rebeca Neves Heinzen. Discipline of Human Structural Anatomy, University of São Paulo School of Medicine, Av. Dr. Arnaldo, \\ 455, São Paulo, SP, Brazil. Email: rebecanh@gmail.com.
}

Backgroundk Nipple-sparing mastectomy (NSM) is increasingly performed for breast cancer (BC) treatment. To ensure local control with this procedure, it is important to obtain clear surgical margins. Here, we aimed to estimate the confidence in intraoperative evaluation of the retroareolar margin (IERM) and the necessity of removing the intra-nipple ducts.

Methods: In this retrospective cohort study, we evaluated 224 BC (infiltrating carcinoma 178, ductal carcinoma in situ 46) patients, who underwent NSM. IERM was determined via cytology and frozen sections. Following gland removal, the intra-nipple ducts were excised and embedded in paraffin for analysis. The retroareolar tissue was also paraffin-embedded and reanalyzed for definitive evaluation of retroareolar margins (DERM). The IERM predictive capacity in relation to DERM and the frequency of intra-nipple duct involvement were estimated.

Results: IERM classified the sub-nipple areolar complex area as cancer-free in 219 cases (97.8\%). The condition of clear retroareolar margin was confirmed by DERM in 216 cases (98.6\%). The IERM accuracy was estimated as $98.6 \%$. Ductal carcinoma in situ was detected in intra-nipple ducts using paraffin sections in $1.8 \%$ of the cases, despite clear IERM (4/219).

Conclusions: In conclusion, IERM affords high accuracy and its results are suitable to manage the nippleareolar complex. Nevertheless, some patients may retain residual disease in the intra-nipple ducts; thus, these ducts should ideally be removed during NSM.

Keywords: Breast cancer (BC); nipple-sparing mastectomy (NSM); surgical margins; nipple ducts

Submitted Mar 27, 2020. Accepted for publication May 20, 2020.

doi: $10.21037 / g s-20-405$

View this article at: http://dx.doi.org/10.21037/gs-20-405 


\section{Introduction}

Nipple-sparing mastectomy (NSM), also termed mammary adenectomy or total skin-sparing mastectomy, is an evolving procedure for patients with breast cancer (BC), which involves resection of all gross visible glandular tissue, while preserving the overlying breast skin envelope and the nipple-areolar complex (NAC). NSM was pioneering used for BC treatment in 1980 by Gentil et al. (1), and recently disseminated by Benediktsson and Perbeck (2) and Gerber et al. (3), who compared NSM with more radical mastectomy among selected patients and observed very satisfactory results. Currently, an increasing body of evidence-based data supports NSM as a valid alternative for the therapy of selected patients with $\mathrm{BC}$ and invasive breast carcinomas (IBC) or ductal carcinomas in situ (DCIS) (4-10).

With proper eligibility criteria for patients undergoing NSM, NAC relapses are very rare, for which the finding of negative retroareolar margin is paramount for oncologic safety (2,3,11-14). Nevertheless, few studies have focused on the reliability of intraoperative evaluation of the retroareolar margin (IERM) in relation to that of the gold standard, paraffin histopathology. Moreover, the requirement of removal of the ducts inside the nipple remains controversial, especially when the retroareolar margin is clear.

Here, we performed a retrospective study with the aim of assessing the confidence afforded by IERM and the value of harvesting the intra-nipple ducts (IND) in the setting of patients with BC managed via NSM.

We present the following article in accordance with the STROBE reporting checklist (available at http://dx. doi. org/10. 21037/gs-20-405).

\section{Methods}

\section{Study design and ethical approval}

This was a retrospective cohort study based on chart reviews of patients treated at a private institution in São Paulo, Brazil (Prof. Alfredo Barros Clinic). The research protocol was approved by the Ethics Committee of the University of São Paulo School of Medicine, which, consider the retrospective analysis of charts, dispensed individual inform consent.

\section{Study population}

Clinical and histopathologic data from patients who fulfilled the criteria for therapeutic NSM indication were collected from January 2007 to December 2019. For all patients, NSM was performed by a single dedicated breast surgeon (ACSD Barros).

Patients were eligible if they met all of the following inclusion criteria: DCIS or early infiltrating carcinoma with the largest tumor focus $\leq 3.0 \mathrm{~cm}$, tumor-nipple distance (TND) $>2.0 \mathrm{~cm}$ by physical examination and magnetic resonance imaging (MRI) performed in a 3-T system, axilla clinically negative or with movable level I-II lymph nodes ( $\mathrm{cN} 0-\mathrm{cN} 1)$, and clear surgical margins as determined by intraoperative evaluation.

Patients were excluded by the presence of at least one of the following conditions: neoadjuvant chemotherapy, clinical evidence of skin/NAC involvement, occult BC, nipple discharge, more than three centers/foci of neoplasia, male $\mathrm{BC}$ and missing data.

\section{NSM protocol}

The most frequent incision employed for NSM was the vertical radial, from the areola to the inframammary fold, elongated by up to $25 \%$ of the areolar circumference into the axillary direction. When a superficial and peripheral neoplasia was present, located $\geq 2.0 \mathrm{~cm}$ from the areolar border and close to the skin ( $\leq 2.0 \mathrm{~cm}$ in depth), an elliptical paddle incision was made in the overlying tumor skin, which may have been extended to the areolar border.

The skin flaps were carefully elevated using a diathermy knife. Cutting within the slim fascia between the subcutaneous fat and the glandular tissue was preferentially adopted. The surgeon left a flap with approximately $0.5 \mathrm{~cm}$ of thickness in the sub-NAC area and 0.5 to $1.0 \mathrm{~cm}$ flap thickness toward the gland periphery. After an "en bloc" resection of the breast parenchyma along the major pectoralis muscle fascia, the surgeon cautiously identified the margins of the specimen, in particular, the retroareolar margin (Figure 1).

\section{Anatomopathological aspects}

During the surgery, the patients were submitted to IERM. The sub-NAC margin was microscopically analyzed by imprint cytology and examination of the whole surface area through frozen sections. Unfixed fragments were placed inside a cryostat and frozen (4- $\mu \mathrm{m}$-thick, at interval of $200 \mu \mathrm{m})$. For each fragment, histologic sections were then stained using hematoxylin-eosin (H\&E) and examined with an optical microscope. For definitive evaluation of the 


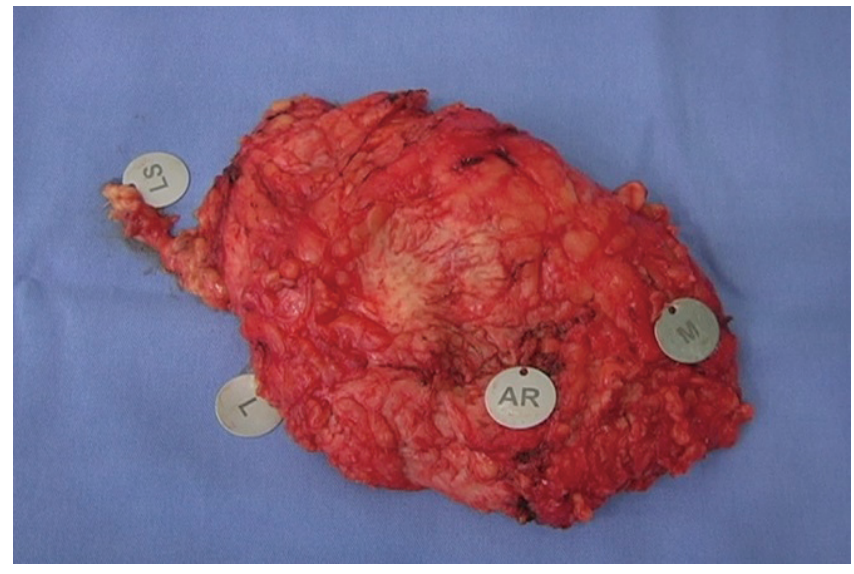

Figure 1 "En bloc" breast parenchyma resection with margin identification. AR, retroareolar margin; L, left margin; $M$, medial margin; LS, sentinel node.

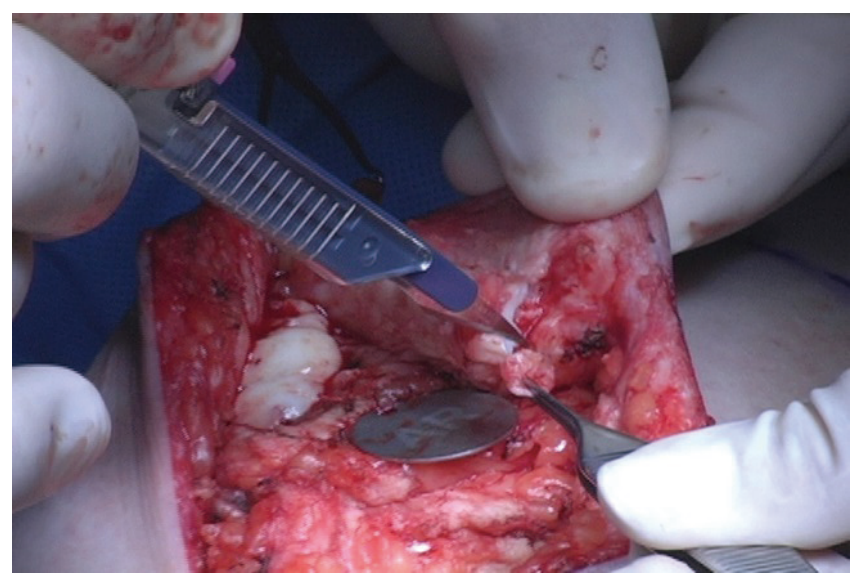

Figure 2 Intra-nipple duct removal. A central bundle was excised with a fine-end knife as a separate specimen. AR, retroareolar margin.

retroareolar margin (DERM), the frozen section fragments were embedded in paraffin and sectioned. The sections (4- $\mu \mathrm{m}$-thick) were stained with $\mathrm{H} \& \mathrm{E}$ and examined. If the margin was negative, the NAC was preserved. The margins were deemed positive if they contain malignant cells (DCIS or IBC). Precursor lesions such as atypical ductal hyperplasia or lobular neoplasia did not warrant a positive margin designation. The NAC was immediately excised when positive IERM results were obtained.

Following gland removal, the nipple was inverted, and the ducts arranged inside in a central bundle were excised with a fine-end knife as a separate specimen, leaving only a thin tissue rim (1.0-2.0 $\mathrm{mm}$ ) under the mammary papilla skin (Figure 2). Subsequently, all patients underwent immediate breast reconstruction via silicone implants placed in a submuscular pocket. Simultaneous mastopexy was performed in women with glandular ptosis, in whom the NAC had migrated and was centralized in the breast mound.

After surgery, the entire specimen was submitted to routine paraffin embedding and reanalyzed microscopically for DERM. IND were solely examined using paraffin sections. Eventual positive findings at the retroareolar margin or at the intra-nipple ducts in the definitive analyses indicated NAC removal in a second-step surgical procedure.

\section{Data analysis}

To evaluate the confidence in IERM, its predictive capacity in relation to that of DERM was estimated based on sensitivity, specificity, predictive positive value, negative predictive value, and accuracy. SPSSV $20^{\circledR}$ was used for the analysis. False negative rates of IERM were calculated first for the whole casuistic, and afterward separately for invasive and ductal in situ cases. Frequency analysis of the intranipple ducts involvement in cases of clear intraoperative or postoperative evaluation of the retroareolar margin was also estimated.

\section{Results}

\section{Subjects and disease characteristics}

A total of 224 patients with cancer-bearing breasts were treated by NSM (178 IBC and 46 DCIS). Mean patient age was 51 years (range, 27-84 years); 119 women were premenopausal (53.1\%) and 105 postmenopausal (46.9\%). Tables 1,2 show the histopathologic and molecular data of the patients.

Among the infiltrating carcinomas, the histopathologic subtypes were as follows: invasive not otherwise specified 137 cases $(77.0 \%)$, invasive lobular carcinoma 27 cases (15.2\%), microinvasive carcinoma 6 cases $(3.4 \%)$, invasive micropapillary carcinoma 4 cases $(2.2 \%)$, tubular carcinoma 2 cases $(1.1 \%)$, metaplastic carcinoma and carcinoma with apocrine differentiation 1 case each $(0.6 \%)$.

The subtypes among the DCIS cases included: cribriform 20 cases $(43.5 \%)$, solid 11 cases (23.9\%), micropapillary 3 cases (6.5\%), clinging 3 cases (6.5\%), papillary 2 cases ( $4.3 \%)$, apocrine 1 case $(2.2 \%)$, and unknown 6 cases. Nuclear grade 
Table 1 Characteristics of the patients with infiltrating carcinoma

\begin{tabular}{|c|c|c|}
\hline Characteristic & $\mathrm{n}$ & $\%$ \\
\hline \multicolumn{3}{|c|}{ Morphologic subtype } \\
\hline Invasive NST & 137 & 77.0 \\
\hline Lobular & 27 & 15.2 \\
\hline Other & 14 & 7.9 \\
\hline \multicolumn{3}{|l|}{ Tumor size } \\
\hline pT1 & 152 & 85.4 \\
\hline рT2 & 26 & 14.6 \\
\hline \multicolumn{3}{|l|}{ Histologic grade } \\
\hline I & 33 & 18.5 \\
\hline II & 79 & 44.4 \\
\hline III & 41 & 23 \\
\hline Unknown & 25 & 14 \\
\hline \multicolumn{3}{|c|}{ Lymph node status } \\
\hline Negative & 135 & 75.8 \\
\hline Positive & 43 & 24.2 \\
\hline \multicolumn{3}{|c|}{ Multicentricity/multifocality } \\
\hline Yes & 60 & 33.7 \\
\hline No & 118 & 66.3 \\
\hline \multicolumn{3}{|c|}{ Estrogen receptor } \\
\hline Positive & 151 & 84.8 \\
\hline Negative & 24 & 13.5 \\
\hline Unknown & 3 & 1.7 \\
\hline \multicolumn{3}{|l|}{ HER-2 } \\
\hline Negative & 29 & 16.3 \\
\hline Positive & 144 & 80.9 \\
\hline Unknown & 5 & 2.8 \\
\hline
\end{tabular}

NST, not otherwise specified.

1: $6.5 \%, 2: 39.1 \%, 3: 45.6 \%$, and unknown: $8.7 \%$.

\section{IERM exbibits high accuracy and specificity}

IERM yielded negative findings in 219 cases (97.8\%). The NAC was immediately removed in 5 cases with malignancy on the ink of the sub-NAC margin. Clearness of the retroareolar margin was confirmed in 216 patients (98.6\%) in the definitive paraffin-embedded tissue sections. In the three cases with late diagnosis of margin involvement, the
Table 2 Characteristics of the patients with ductal carcinoma in situ

\begin{tabular}{lcc}
\hline Characteristic & $\mathrm{n}$ & $\%$ \\
\hline Comedo & 24 & 52.2 \\
Yes & 15 & 32.6 \\
No & 7 & 15.2 \\
Unknown & & \\
Nuclear grade & 3 & 6.5 \\
1 & 18 & 39.1 \\
2 & 21 & 45.7 \\
3 & 4 & 8.7 \\
Unknown & & \\
Multicentricity/multifocality & 25 & 54.3 \\
Yes & 16 & 34.8 \\
No & 5 & 10.9 \\
Unknown & & \\
Estrogen receptor & & 76.1 \\
Positive & 35 & 6.5 \\
Negative & 3 & 17.4 \\
Unknown & 8 & \\
\hline
\end{tabular}

Comedo, comedonecrosis.

NAC was also withdrawn.

Table 3 illustrates intraoperative and definitive results of the retroareolar margin evaluation in the whole sample. Overall, $2.2 \%$ of the patients showed a positive margin by IERM and $3.6 \%$ by paraffin sections.

The predictive capacity parameters of IERM in relation to those of DERM are outlined in Table 4, revealing its good performance with high accuracy (98.6\%) maximal specificity (100\%), and moderate sensitivity (62.5\%). Figures 3,4 show clean and involved retroareolar margins on paraffin sections.

\section{Low frequency of positive IND even with clear IERM and DERM}

Four cases of DCIS lesions (Figure 5) were found in the IND examined as paraffin sections in 219 cases with negative IERM (1.8\%). All lesions occurred in IBC cases (4/175). No case of malignancy inside the nippleducts was detected among the 44 patients with DCIS. Alternatively, when the three cases of patients exhibiting 
Table 3 Results of retroareolar margin evaluation in intraoperative and definitive analysis

\begin{tabular}{lcccc}
\hline \multirow{2}{*}{ Cases } & \multicolumn{2}{c}{ Intraoperative } & \multicolumn{2}{c}{ Definitive } \\
\cline { 2 - 5 } & $\mathrm{n}$ & $\%$ & $\mathrm{n}$ & $\%$ \\
\hline Infiltrating carcinoma & & & & \\
$\quad$ Clear & 175 & 98.3 & 172 & 96.6 \\
Involved & 3 & 1.7 & 6 & 3.4 \\
Ductal carcinoma in situ & & & & \\
$\quad$ Clear & 44 & 95.6 & 44 & 95.7 \\
$\quad$ Involved & 2 & 4.3 & 2 & 4.3 \\
Total & & & & \\
$\quad$ Clear & 219 & 97.8 & 216 & 96.4 \\
Involved & 5 & 2.2 & 8 & 3.6 \\
\hline
\end{tabular}

Table 4 Predictive capacity of intraoperative evaluation of retroareolar margin in relation to definitive analysis

\begin{tabular}{lccc}
\hline Attribute & $\begin{array}{c}\text { Infiltrating cases } \\
(\%)\end{array}$ & $\begin{array}{c}\text { Ductal in situ } \\
\text { cases (\%) }\end{array}$ & Total (\%) \\
\hline Sensitivity & 50 & 100 & 62.5 \\
Specificity & 100 & 100 & 100 \\
$\begin{array}{l}\text { Positive predictive } \\
\text { value }\end{array}$ & 100 & 100 & 100 \\
$\begin{array}{l}\text { Negative predictive } \\
\text { value }\end{array}$ & 98.3 & 100 & 98.6 \\
Accuracy & 98.3 & 100 & 98.6 \\
\hline
\end{tabular}

involved retroareolar margin in the definitive examination were excluded, the frequency of affected IND halved to $0.9 \%(2 / 216)$. Moreover, in two cases with positive IND, the margin was considered clear by IERM and involved by DERM (Table 5). Ultimately, NAC was removed in 10 of 224 cases (4.5\%), of which 5 were indicated by IERM, 3 by DERM, and 2 by nipple-duct involvement after clear IERM and DERM.

\section{Discussion}

A paradigm shift from radical surgery toward less aggressive personalized procedures has evolved over the last decades, with NSM being increasingly performed for patients with BC without evidence of NAC invasion, with acceptable safety $(7,8,10-12)$. For example, Wu et al. (12) reported a

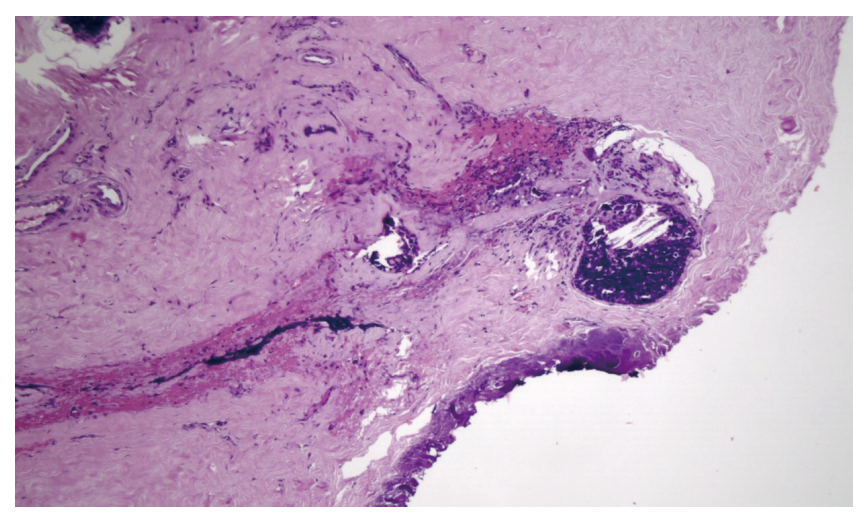

Figure 3 Clear retroareolar margin, paraffin block, perpendicular section (HE, 100x).

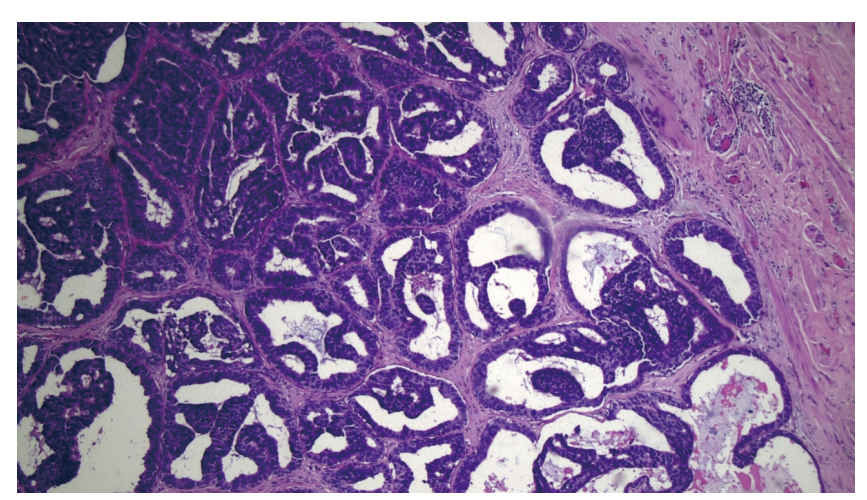

Figure 4 Involved retroareolar margin, paraffin block, tangential section (HE, 100x).

5 -year cumulative incidence of cancer recurrence at the NAC of $3.5 \%$; moreover, our group did not identify any NAC recurrence among $161 \mathrm{NSMs}$ for BC treatment (7).

NAC preservation is very important for women's satisfaction with their breast silhouette; thus, NSM provides psychologic benefits for the patients as the NAC has strong symbolic and psychologic significance (15). However, although it may therefore be tempting for surgeons to offer NSM for BC treatment, NAC-sparing surgeries should be recommended with caution owing to concerns regarding proper case selection and oncologic outcomes $(7,8,16,17)$.

We consider NSM a suitable alternative for women who are opting for "maximal surgery" instead of breastconserving surgeries, mainly in cases associated with one or more of the following conditions: hereditary BC, young age, tumor multifocality/multicentricity, suspicious diffuse microcalcifications, difficulty in achieving clear margins, or 


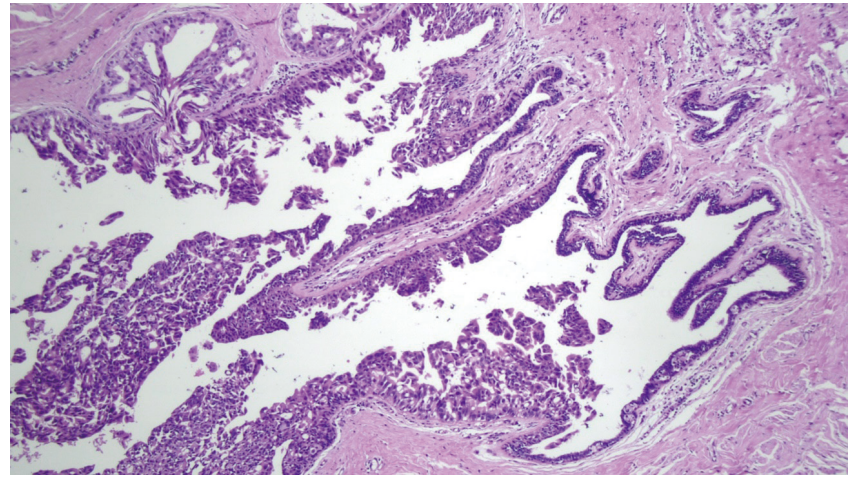

Figure 5 Carcinoma ductal in situ in an intra-nipple duct (HE, $100 \times)$.

Table 5 Frequency of ductal carcinoma in situ in the intra-nipple ducts following verification of the clear retroareolar margin

\begin{tabular}{lcc}
\hline \multirow{2}{*}{ Examination } & \multicolumn{2}{c}{ Positive nipple ducts } \\
\cline { 2 - 3 } & $\mathrm{n}$ & $\%$ \\
\hline Intraoperative & 4 & 1.8 \\
Definitive & 2 & 0.9 \\
\hline
\end{tabular}

contraindication for radiotherapy. In comparison, Coopey and Smith (18) specify that absolute contraindications for NSM include clinical or imaging of direct NAC infiltration, or involvement of the sub-nipple margin observed on pathology. Nevertheless, a tendency exists to increase the allowed tumor size for case selection up to $5.0 \mathrm{~cm}$ and at some institutions, locally advanced BC successfully managed by neoadjuvant chemotherapy is also included $(17,19,20)$.

As NSM gains popularity, the dilemma of prerequisites for this surgery becomes more relevant. At our institution, we have followed stringent criteria with one condition for preserving the NAC being a safe TND that could be reliably measured by MRI $(21,22)$. For the present study, our inclusion criteria entailed a TND $\geq 2.0 \mathrm{~cm}$ as estimated by this imaging method. Signs of nipple involvement by MRI include periareolar skin thickening, enhancement of the ipsilateral nipple, and a short TND. Several cut-off values of TND have been suggested, ranging from 0.5 to $2.0 \mathrm{~cm}$ $(23,24)$. However, Piato et al. estimated a sensitivity of $29.7 \%$ and specificity of $97.7 \%$ for MRI in terms of NAC neoplastic involvement, indicating that although MRI is a useful tool, retroareolar margin examination is mandatory (21).

The task of the surgeon, to remove a maximum amount of breast tissue while achieving low morbidity, and the best possible cosmetic results, is not simple. Papassotiropoulos et al. (25) performed biopsies (at least 10) at pre-established points from the skin envelope after breast removal in NSM and skin-sparing mastectomy. They found at least one focus of residual breast tissue in $51.3 \%$ of the patients and estimated the median residual breast tissue percentage per breast as $7.1 \%$, mainly in the central area (25). Notably, they observed that highly experienced surgeons can performed NSM more radically with a low rate of skin flap necrosis. The eventual permanence of the extent of undesirable residual breast tissue following NSM can be identified by MRI. In this manner, the acquisition of an imaging control after surgery is meaningful for guiding additional surveillance in addition to therapeutic intervention, including radiation therapy (26).

Notably, the indication of complementary radiotherapy is controversial following NSM with thin flaps and free margins. The majority of patients treated accordingly do not require adjuvant irradiation, which is generally only recommended in cases with more than three affected lymph nodes or when early postoperative imaging shows an excess of remaining tissue (27). Specifically, although radiotherapy is efficient, it frequently causes adverse effects including capsular contracture, contour asymmetry, and dermatitis.

To ensure oncologic safety following NSM, it is considered important to avoid remnant neoplastic cells beneath the NAC. Toward this end, intraoperative microscopic analysis of the sub-nipple tissue constitutes a valuable strategy. Our findings provided evidence demonstrating the high accuracy of IERM in the patient cohort (98.6\%) in addition to within the IBC $(98.3 \%)$ and (100\%) DCIS subgroups. The predictive parameter of IERM in relation to that of the paraffin exams thus allowed us to ascertain that during surgery, a retroareolar en-face margin microscopic analysis should be performed in all cases of NAC-sparing procedures.

In comparison, the validity of sub-nipple frozen sections was analyzed by Duarte et al. (28) who conducted a prospective $e x$ vivo study with 68 patients with BC that had undergone nipple-sacrificing surgeries. The sub-nipple area was dissected immediately after the surgery, simulating an NSM flap. The retroareolar tissue was submitted to frozen sectioning, imprint cytology, and paraffin sectioning. The nipples were examined separately via permanent histology as a gold standard parameter, with eight cases showing occult involvement. 
The accuracy of the frozen sections was $86.8 \%$, that of cytology $76.5 \%$, and paraffin $86.8 \%$. False negative rates were, respectively, $6.8 \%, 9.3 \%$, and $5.3 \%$, for frozen sections, cytology, and definitive histopathology (28). Alternatively, Morales Piato et al., using the same study model, found an accuracy of $95.4 \%$ for frozen section exams (29).

The aim of sub-nipple microscopic analysis is to preserve the NAC with a minimal chance of leaving residual malignancy in this area. Our results indicated that IERM facilitated intraoperative decision-making and facilitated earlier recognition of occult tumor cells in the NAC, avoiding two-stage procedures. Moreover, although some difficulties are associated with freezing artifacts and interpretation, several publications favored IERM in daily practice $(2,10,30,31)$. The first report regarding the predictive capacity of IERM, by Luo et al. (30) involving $52 \mathrm{NSM}$ cases, indicated a predictive positivity value of $100 \%$, negative predictive value of $83 \%$, sensitivity of $38 \%$, and specificity of $100 \%$. In addition, Alperovich et al. (31) found that among 307 cases of NSM with subnipple margin assessment, 12 biopsies were positive with permanent paraffin sections (3.9\%). Of these, five were shown to be false negative intraoperatively, resulting in 58\% and $100 \%$ IERM sensitivity and specificity, respectively (31). Furthermore, in the classical paper published by Benediktsson and Perbeck (2), including 216 breasts, IERM sensitivity was $90.9 \%$ and specificity was $98.5 \%$.

Notably, IND removal is not likely to affect nipple viability following NSM. The small ducts inside the nipple are arranged in a central bundle and this configuration allows their ready excision. In addition, microvessel counts inside the central duct bundle of the mammary papilla have been compared with those of the peripheral rim by Rusby et al. (32) using cross sections through a nipple stained with anti-factor VIII antibody. They perceived that a $2-\mathrm{mm}$ rim of peripheral nipple tissue allows the complete removal of the ductal bundle in $96 \%$ of the sections and retained $50 \%$ of the vessels, whereas only $29 \%$ of vessels are removed with the duct bundle. For these authors, when the central vessels of the nipple are excised, the remaining peripheral rim vessels provide sufficient vascular supply to maintain a viable nipple (32). Moreover, Smith and Coopey (33) stated that it is possible for a skillful surgeon to lift off tissue from within the nipple, exposing its underside dermis and leaving only a thin layer of local tissue $(2.0 \mathrm{~mm})$ under the nipple skin without markedly increasing the rate of nipple necrosis. However, exceptions may occur in patients with diabetes or heavy smokers with damaged microvasculature, and in women with obesity and large ptotic breasts (33). In particular, the preservation of the perforator vascular branches that derive from the second and third internal thoracic vessels, emerging at the lateral sternum border, is of utmost importance to prevent nipple ischemia (7). For this reason, medial incisions should be preferentially avoided.

In the present study, we did not observe any case of invasive carcinoma in the IND, although four cases of DCIS were diagnosed despite negative findings for the margins reported by IERM. In two of these cases both exams for retroareolar margins (IERM and definitive) were negative. Therefore, we consider that the removal of the IND is advisable in cases where almost complete excision of ductal epithelium is desired, which mainly occur when radiotherapy is being avoided.

Few previous studies have demonstrated the involvement of intrapapillary ducts when the retroareolar margin is clear. Tramm et al. (34) reported a case of DCIS in the nipple with clear NAC base sections, which they termed a "skip lesion". A prospective study conducted by Crowe et al. (35) involving $86 \mathrm{NSMs}$ performed for BC treatment identified IND involvement in $9(11 \%)$ cases, including just one case of IBC. However, the authors preferred to limit NSM to patients whose tumors measured $\leq 3.5 \mathrm{~cm}$ as determined by careful imaging assessment (35).

The retroareolar en-face margin may be used to test for occult nipple involvement in patients undergoing NACsparing surgeries; however, we consider that this approach may offer limited precision. Previously, Brachtel et al. (36) observed that in $20 \%$ of the cases of nipple involvement following therapeutic mastectomies, the sub-nipple margin appeared clear (false negative). More recently, Cont et al. (37) recommended double intraoperative evaluation to detect additional positive cases, as they found that subareolar duct and IND removal had a higher predictive value than that of the subareolar exam alone, even when using the criterium of TND $\geq 5.0 \mathrm{~mm}$ preoperatively by MRI. Our results supported a similar conclusion.

Our recommendation for patients exhibiting a positive subareolar margin by means of IERM or paraffin sections is that their NAC be excised. When a skipped neoplastic progression to IND is observed despite a clear retroareolar margin, we advocate a second surgery to remove the whole NAC or at least to excise the nipple with a $2-\mathrm{mm}$ rim of 
areola.

This study was not without limitations and further research is warranted to clarify important practical aspects. For example, as intraoperative analysis of the IND was not performed, we could not determine its feasibility and efficiency. In addition, we did not assess the outcome of conservation of the nipple with positive ducts when the IND are positive, yet the intra nipple margins are clear.

In summary, our data indicated that sub-NAC area involvement is rare in well selected patients with BC treated by NSM, and that IERM affords high accuracy to predict retroareolar margin involvement. Nevertheless, even in cases of clear margins, we found that some patients could exhibit residual disease in the IND and the ducts should ideally be removed during NSM.

\section{Acknowledgments}

Funding: None.

\section{Footnote}

Reporting Checklist: The authors have completed the STROBE reporting checklist. Available at http://dx.doi. org/10.21037/gs-20-405

Data Sharing Statement: Available at http://dx.doi. org/10.21037/gs-20-405

Conflicts of Interest: All authors have completed the ICMJE uniform disclosure form (available at http://dx.doi. org/10.21037/gs-20-405). The authors have no conflicts of interest to declare.

Ethical Statement: The authors are accountable for all aspects of the work in ensuring that questions related to the accuracy or integrity of any part of the work are appropriately investigated and resolved. Rebeca N. Heinzen is the guarantor of this work and, as such, had full access to all of the data in the study and takes responsibility for the integrity of the data and the accuracy of the data analysis. This was a retrospective cohort study based on chart reviews of patients treated at a private institution in São Paulo, Brazil (Prof. Alfredo Barros Clinic). The research protocol was approved by the Ethics Committee of the University of São Paulo School of Medicine, which, consider the retrospective analysis of charts, dispensed individual inform consent.

Open Access Statement: This is an Open Access article distributed in accordance with the Creative Commons Attribution-NonCommercial-NoDerivs 4.0 International License (CC BY-NC-ND 4.0), which permits the noncommercial replication and distribution of the article with the strict proviso that no changes or edits are made and the original work is properly cited (including links to both the formal publication through the relevant DOI and the license). See: https://creativecommons.org/licenses/by-nc-nd/4.0/.

\section{References}

1. Gentil F, Callia W, de Souza e Sá A, et al. Mammary carcinoma--multidisciplinary treatment with bilateral mastectomy and immediate reconstruction. J Surg Oncol 1980;14:173-93.

2. Benediktsson KP, Perbeck L. Survival in Breast Cancer after Nipple-Sparing Subcutaneous Mastectomy and Immediate Reconstruction with Implants: A Prospective Trial with 13 Years Median Follow-Up in 216 Patients. Eur J Surg Oncol 2008;34:143-8.

3. Gerber B, Krause A, Dieterich M, et al. The Oncological Safety of Skin Sparing Mastectomy with Conservation of the Nipple-Areola Complex and Autologous Reconstruction: An Extended Follow-Up Study. Ann Surg 2009;249:461-8.

4. Dinner MI, Labandter HP. Total Mammary Adenectomy with Histologic Evaluation and Immediate Reconstruction. Plast Reconstr Surg 1981;68:505-11.

5. Mitchell SD, Willey SC, Beitsch P, et al. Evidence Based Outcomes of the American Society of Breast Surgeons Nipple Sparing Mastectomy Registry. Gland Surg 2018;7:247-57.

6. Mallon P, Feron JG, Couturaud B, et al. The Role of Nipple-Sparing Mastectomy in Breast Cancer: A Comprehensive Review of the Literature. Plast Reconstr Surg 2013;131:969-84.

7. Barros ACSD, Carvalho HA, Andrade FEM, et al. Mammary Adenectomy Followed By Immediate Reconstruction For Treatment Of Patients With EarlyInfiltrating Breast Carcinoma: A Cohort Study. São Paulo Med J 2019;137:336-42.

8. Smith BL, Tang R, Rai U, et al. Oncologic Safety of 
Nipple-Sparing Mastectomy in Women with Breast Cancer. J Am Coll Surg 2017;225:361-5.

9. Petit JY, Veronesi U, Orecchia R, et al. NippleSparing Mastectomy with Nipple Areola Intraoperative Radiotherapy: One Thousand and One Cases of a Five Years Experience at the European Institute of Oncology of Milan (EIO). Breast Cancer Res Treat 2009;117:333-8.

10. de Alcantara Filho P, Capko D, Barry JM, et al. NippleSparing Mastectomy for Breast Cancer and Risk-Reducing Surgery: The Memorial Sloan-Kettering Cancer Center Experience. Ann Surg Oncol 2011;18:3117-22.

11. Orzalesi L, Casella D, Santi C, et al. Nipple Sparing Mastectomy: Surgical and Oncological Outcomes from a National Multicentric Registry with 913 Patients (1006 Cases) over a Six Year Period. Breast 2016;25:75-81.

12. Wu ZY, Kim HJ, Lee JW, et al. Breast Cancer Recurrence in the Nipple-Areola Complex after Nipple-Sparing Mastectomy with Immediate Breast Reconstruction for Invasive Breast Cancer. JAMA Surg 2019;154:1030-7.

13. Tang R, Coopey SB, Merrill A, et al. Positive Nipple Margins in Nipple-Sparing Mastectomies: Rates, Management, and Oncologic Safety. J Am Coll Surg 2016;222:1149-55.

14. Amara D, Peled AW, Wang F, et al. Tumor Involvement of the Nipple in Total Skin-Sparing Mastectomy: Strategies for Management. Ann Surg Oncol 2015;22:3803-8.

15. Wei CH, Scott AM, Price AN, et al. Psychosocial and Sexual Well-Being Following Nipple-Sparing Mastectomy and Reconstruction. Breast J 2016;22:10-7.

16. Benson JR, Dumitru D, Malata CM. Oncologic Safety of Conservative Mastectomy in the Therapeutic Setting. Gland Surg 2016;5:37-46.

17. Young WA, Degnim AC, Hoskin TL, et al. Outcomes of > 1300 Nipple-Sparing Mastectomies with Immediate Reconstruction: the Impact of Expanding Indications on Complications. Ann Surg Oncol 2019;26:3115-23.

18. Coopey SB, Smith BL. The Nipple is Just Another Margin. Ann Surg Oncol 2015;22:3764-6.

19. Wang M, Huang J, Chagpar AB. Can I Keep My Nipple? Factors Influencing the Surgical Decision between SkinSparing and Nipple-Sparing Mastectomy. Am Surg 2019;85:768-71.

20. Bartholomew AJ, Dervishaj OA, Sosin M, et al. Neoadjuvant Chemotherapy and Nipple-Sparing Mastectomy: Timing and Postoperative Complications. Ann Surg Oncol 2019;26:2768-72.

21. Piato JR, de Andrade RD, Chala LF, et al. MRI to Predict Nipple Involvement in Breast Cancer Patients. AJR Am J
Roentgenol 2016;206:1124-30.

22. Frey JD, Salibian AA, Lee J, et al. Oncologic trends, outcomes, and risk factors for locoregional recurrence: an analysis of tumor-to-nipple distance and critical factors in therapeutic nipple-sparing mastectomy. Plast Reconstr Surg 2019;143:1575-85.

23. Mariscotti G, Durando M, Houssami N, et al. Preoperative MRI Evaluation of Lesion-Nipple Distance in Breast Cancer Patients: Thresholds for Predicting Occult Nipple-Areola Complex Involvement. Clin Radiol 2018;73:735-43.

24. Machida Y, Shimauchi A, Igarashi T, et al. Reproducibility and Significance of Findings Relevant to Nipple-Areolar Complex Involvement. Breast Cancer 2018;25:456-63.

25. Papassotiropoulos B, Güth U, Chiesa F, et al. Prospective Evaluation of Residual Breast Tissue after Skin-or NippleSparing Mastectomy: Results of the SKINI-Trial. Ann Surg Oncol 2019;26:1254-62.

26. Giannotti DG, Hanna SA, Cerri GG, et al. Analysis of Skin Flap Thickness and Residual Breast Tissue after Mastectomy. Int J Radiat Oncol Biol Phys 2018;102:82-91.

27. Marta GN, Poortmans P, de Barros AC, et al. Multidisciplinary International Survey of Post-Operative Radiation Therapy Practices after Nipple-Sparing or SkinSparing Mastectomy. Eur J Surg Oncol 2017;43:2036-43.

28. Duarte GM, Tomazini MV, Oliveira A, et al. Accuracy of Frozen Section, Imprint Cytology, and Permanent Histology of Sub-Nipple Tissue for Predicting Occult Nipple Involvement in Patients with Breast Carcinoma. Breast Cancer Res Treat 2015;153:557-63.

29. Morales Piato JR, Aguiar FN, Mota BS, et al. Improved Frozen Section Examination of the Retroareolar Margin for Prediction of Nipple Involvement in Breast Cancer. Eur J Surg Oncol 2015;41:986-90.

30. Luo D, Ha J, Latham B, et al. The Accuracy of Intraoperative Subareolar Frozen Section in NippleSparing Mastectomies. Ochsner J 2010;10:188-92.

31. Alperovich M, Choi M, Karp NS, et al. Nipple-sparing Mastectomy and Sub-areolar Biopsy: To Freeze or not to Freeze? Evaluating the Role of Sub-areolar Intraoperative Frozen Section. Breast J 2016;22:18-23.

32. Rusby JE, Brachtel EF, Taghian A, et al. Implications for Nipple-Sparing Mastectomy. Am J Surg 2007;194:433-7.

33. Smith BL, Coopey SB. Nipple-Sparing Mastectomy. Adv Surg 2018;52:113-26.

34. Tramm T, Zuckerman K, Tavassoli FA. Skip Lesion of DIN (DCIS) in the Nipple in a Case of Breast Cancer. Int J Surg Pathol 2011;19:817-21. 
35. Crowe JP, Patrick RJ, Yetman RJ, et al. NippleSparing Mastectomy Update: One Hundred FortyNine Procedures and Clinical Outcomes. Arch Surg 2008;143:1106-10.

36. Brachtel EF, Rusby JE, Michaelson JS, et al.

Clinicopathologic Findings in 316 Consecutive

Cite this article as: Heinzen RN, Barros ACSD, Carvalho FM, Aguiar FN, Nimir CCBA, Jacomo AL. Nipple-sparing mastectomy for early breast cancer: the importance of intraoperative evaluation of retroareolar margins and intra-nipple duct removal. Gland Surg 2020;9(3):637-646. doi: 10.21037/gs$20-405$
Mastectomy Specimens. J Clin Oncol 2009;27:4948-54.

37. Cont NT, Maggiorotto F, Martincich L, et al. Primary Tumor Location Predicts the Site of Local Relapse after Nipple-Areola Complex (NAC) Sparing Mastectomy. Breast Cancer Res Treat 2017;165:85-95. 